\title{
Multidisciplinary approach in the rehabilitation of congenitally missing lateral incisors: a new trend in daily practice
}

\author{
Nezar Watted ${ }^{1}$, Péter Borbély², Ali Watted ${ }^{3}$, Ghannam Nidal ${ }^{4}$, Abdulgani Azzaldeen ${ }^{5}$ and Muhamad Abu-Hussein ${ }^{* 6}$ \\ ${ }^{1}$ UUniversity Hospital of Würzburg Clinics and Policlinics for Dental, Oral and Maxillofacial Diseases of the Bavarian Jul ius-Maximilian-University Wuerzburg, \\ Germany and Department of Orthodontics, Arab American University, Jenin, Palestine \\ 2Department of Orthodontics, University of Debrecen, Hungary \\ ${ }^{3}$ University Hospital of Regensburg, dental school, University of Regensburg, Germany \\ ${ }^{4}$ Arab American University, Jenin, Palestine \\ ${ }^{5}$ Department of Conservative Dentistry, Al-Quds University, Jerusalem, Palestine \\ ${ }^{6}$ Department of Pediatric Dentistry, University of Athens, Greece
}

\begin{abstract}
Agenesis, the absence of permanent teeth, is a common occurrence among dental patients. The total incidence of tooth agenesis is about $4.2 \%$ among patients that are seeking orthodontic treatment and with the exception of third molars, the maxillary lateral incisors are the most common congenitally missing teeth with about a $2 \%$ incidence. The maxillary lateral incisor is the second most common congenitally absent tooth. There are several treatment options for replacing the missing maxillary lateral incisor, including canine substitution, tooth-supported restoration, or single-tooth implant. Dental implants are an appropriate treatment option for replacing missing maxillary lateral incisor teeth in adolescents when their dental and skeletal development is complete. This case report presents the treatment of a patient with congenitally missing maxillary lateral incisor using dental implants. The paper discusses the aspects of pre-prosthetic orthodontic diagnosis and the treatment that needs to be considered with conservative and fixed prosthetic replacement.
\end{abstract}

\section{Introduction}

Maxillary lateral incisor agenesis is the most common congenitally missing permanent tooth condition in the maxillary anterior region (esthetic zone), representing approximately $20 \%$ of all dental anomalies [1-3]. It has been found to be more prevalent in females [2,4,5], and bilateral MLIA are more frequently reported than unilateral cases [6]. Largely, dental agenesis has been attributed to genetic factors [7], but they may also be caused by environmental factors such as dentoalveolar traumas [8], or radiation therapy [3,4]. The susceptibility of maxillary lateral incisors to dental agenesis has been associated with their anatomical position in the maxillary arch and also the fact that they are the last teeth to develop in their respective classes [3].

Hypodontia usually has a genetic basis and often a high proportion of affected individuals have a family history of hypodontia or associated dental anomalies. Mutation in transcription factors MSX1, PAX9 and AXIN 2 have been identified in families with an autosomal dominant oligodontia. Normally, teeth which are "end of series" are more commonly absent, i.e. lateral incisors, second premolars and third molars. Hypodontia is also often seen in patients presenting with syndromes such as ectodermal dysplasia, Down's syndrome and hemifacial microsomia and in nonsyndromic conditions such as cleft lip and palate. However, familial hypodontia is complex and multifactorial; influenced by a combination of gene function, environmental interaction and developmental timing [9].

To close or not to close; which is better? This is the age old question that orthodontists have been trying to answer for years when treatment planning patients who are missing maxillary lateral incisors [1,2]. A significant number of people in the population are congenitally missing permanent maxillary lateral incisors [1-5]. The demand for orthodontic treatment by these people is high because of the obvious impact that this condition has on both dental and facial esthetics. This is a challenging situation that every orthodontist will encounter on a regular basis [3,4].

There are multiple options when treatment planning these patients. One option is to close the lateral incisor space by moving the canine until it is adjacent to the central incisor and then reshaping it to look like the lateral incisor through a process called canine substitution [6]. The other option is to place the canine at its natural position within the dental arch, filling the void left by the missing lateral incisor with either a single-tooth implant or a tooth-supported restoration $[2,4,6]$.

A recent study by Rendon et al. found that when the permanent

Correspondence to: Abu-Hussein Muhamad, DDS, MScD, MSc, M Dent Sci (Paed Dent), FICD, 123 Argus Street, 10441 Athens, Greece, E-mail: abuhusseinmuhamad@gmail.com

Key words: Congenitally missing teeth, orthodontic space opening, preprosthetic orthodontics, dental implant

Received: December 01, 2016; Accepted: December 23, 2016; Published: December 27, 2016 
maxillary lateral incisor was missing, the canine erupted in a more mesial position within the dental arch closer to the midalveolar plane [7] Araujo also suggests that in patients with congenitally missing maxillary lateral incisors, canines frequently show a mesial pattern of eruption, with a final position in the dental arch that is adjacent and parallel to the central incisors, and that such a condition favors canine substitution [8].

One of the most challenging problems in dentistry is the treatment option for replacement of one or more maxillary lateral incisors that have been lost as a result of traumatic injuries or congenitally missing [5]. Age, location, space limitations, alveolar ridge deficiencies, uneven gingival margins, occlusion, and periodontal factors often necessitate an interdisciplinary approach $[6,7]$. Thus the management of maxillary lateral incisor agenesis needs multiple dental specialties like orthodontics, oral surgery, Periodontics and Prosthodontics [1].

In patients that are congenitally missing maxillary lateral incisors, one more criteria to consider that isn't mentioned in the literature is the gingival margin of the maxillary canine that erupts into the space normally occupied by the maxillary lateral incisor, and its relationship to the gingival margin of the maxillary central incisor. Is the relationship between the gingival margins like a normal maxillary central incisor/ maxillary canine relationship, where the gingival margins are at the same level? Or, is the relationship between the gingival margins of the maxillary central incisor and canine more like a maxillary central incisor/maxillary lateral incisor relationship, where the lateral incisor gingival margin in more incisal than the gingival margin of the maxillary central incisor $[1-6,9]$ ?

Multiple studies have tried to determine what the most esthetic gingival margin relationship is between a maxillary central incisor and a maxillary lateral incisor. This was accomplished by using smiling photographs of the same smile in which only the gingival margin relationship between the maxillary central and lateral incisors had been altered in certain increments. Lay people, dentists, and dental specialists were then asked which photographs they thought were the most esthetic [10-12].

Other studies tried to determine what the most common relationship was between maxillary central incisors and maxillary lateral incisors in the dental arch. This was accomplished by obtaining measurements directly from plaster model casts using digital calipers and from digital photographs of model casts using computer software $[11,13]$.

In general, the treatment options include space maintenance or later incisor rehabilitation with prostheses, dental implants, or orthodontic space closure with camouflaging the maxillary canine to resemble the appearance of a lateral incisor $[1,2]$. The prominence of the canine root eminence is another esthetic consideration of the space closure approach in patients with high smile lines [1]. When space opening is indicated, both orthodontist and prosthodontist perform a key role in determining and establishing space requirements $[4,5]$. The restorative approaches can be divided into two categories (single tooth implant, and tooth supported restorations) where dental implants are the most commonly used to rehabilitate congenitally missing maxillary lateral incisors once skeletal maturity has been reached. When dental implants are contra-indicated, there are mainly three available options: removable partial denture, resin bonded bridge which is a minimally invasive option for rehabilitation of congenitally missing lateral incisor, and full coverage fixed partial denture [1-6].
The term "team approach" has been used throughout the health care industry, and as technologies continue to advance, this term has evolved from simply referring patient back and forth to detailed treatment planning and case selection. In this case report, the restorative dentist presence and participation at stage I surgery was a valuable asset to achieving the ideal esthetic and functional result for this patient $[1-3,5]$.

Ultimately, it is the team approach that accounts for the esthetic and functional success of this case, taking advantage of the synergy of working together to maximize each clinician's skill in contributing to the best, most predictable, least invasive outcome for the patient $[1,3]$.

The paper discusses the aspects of pre-prosthetic orthodontic diagnosis and the treatment that needs to be considered with conservative and fixed prosthetic replacement.

\section{Orthodontic approach}

The orthodontic approach is, in the authors' opinion, the most conservative approach and is favorable if a patient meets certain requirements. These requirements are (1) malocclusion and (2) size, shape, and color of the canines (Figure 1).

According to Kokich et al. there are two malocclusions that permit canine substitution (canine repositioning at the site of the lateral incisor). These are an Angle class II malocclusion, with no crowding in the mandibular arch, or an Angle class I malocclusion, with severe crowding in the lower arch where it is necessary to execute extractions. The final occlusion of both variants should end up in an anterior group function in lateral excursive movements [10]. When evaluating the size, shape, and color of the canine carefully, it can be predicted if recontouring alone is enough for an esthetic result or if the orthodontic treatment has to be combined with subsequent restorative treatment. The ideal canine for substitution has similar proportions in width and convexity as the lateral it should replace. Also, the color should be similar to the color of the central incisor. To establish the proper width, either contralateral incisor may be taken as a reference or some proportional calculations can be made. The authors favors Chu's [14] approach with the formula;

Central incisor $=$ width in $\mathrm{mm}=\mathrm{X}$

Lateral incisor $5=\mathrm{X}-2 \mathrm{~mm}$

Canine $=\mathrm{X}-1 \mathrm{~mm}$

According to this formula by $\mathrm{Chu}$, [14] the canine that substitutes the lateral is approximately $1 \mathrm{~mm}$ too wide. That means that $0.5 \mathrm{~mm}$ of recontouring mesially and distally would have to be done to get the desired width. The convexity of the lateral incisor is more subtle than the convexity of the canine. Also, the canine normally shows in two planes mesiodistally whereas the lateral incisor has just one. It is also necessary to reshape the lingual surface of the canine to achieve a proper overjet and overbite relation, and the cusp tip of the canine needs enameloplasty as well.

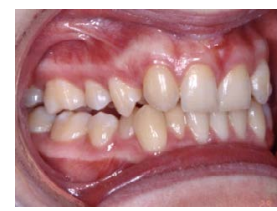

$a$

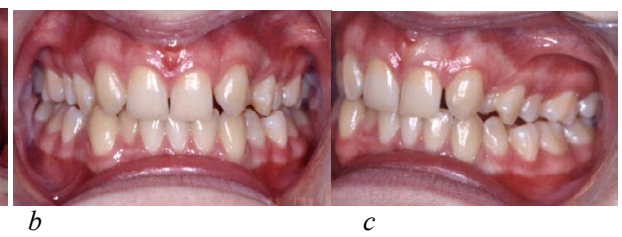

Figure 1a-c. male aged $11 \mathrm{y} 3 \mathrm{~m}$, comes at our observation completion of permanent eruption, showing upper cuspid just in lateral incisors position. 
If all this recontouring requires a significant amount of reduction of enamel, problems may occur. One of the problems that can be faced is that a patient experiences dental hypersensitivity, although Zacchrison et al. has shown that if all these reductions are performed using diamond instruments with abundant water spray cooling on young teeth, there are no long-term changes in tooth sensitivity. If dentin is exposed, an adjunctive restoration may be necessary [15] (Figure 2).

Another problem that occurs is the color of the canine. Usually the canines are one to two shades darker than the central incisor. This problem can get even worse if a lot of reduction has to be performed to flatten a canine with a prominent labial convexity. As the enamel of the canine becomes thinner, the dentin starts to show through the translucent enamel and as a result the tooth appears even darker. One solution for the difference in color is single tooth bleaching, but with thinning the enamel the risk of sensitivity after bleaching increases [15]. Another option is adjunctive restorative treatment (Figure 3).

The bracket placement in canine substitution cases is different. The bracket is not placed with the incisal edge of the canine as a reference but with the gingival margin as the guide. The gingival zeniths of the lateral incisors should be 0.5 to $1 \mathrm{~mm}$ lower than the central incisors, so the canine bracket has to be placed accordingly [15]. To make the final decision if a patient is suitable for the orthodontic approach and to anticipate if additional restorations may be necessary, the authors strongly suggest a carefully executed treatment plan (Figures 4 and 5).

\section{Advantages}

The major advantage of space closure is the permanence of the finished result. This is a one- shot therapy, which means that the overall treatment can be completed by the end of orthodontic treatment at an early age with a permanent result and long-term stability. The alveolar bone height in the actual region is maintained by the early mesial movement of the canine. The individual keeps his natural dentition

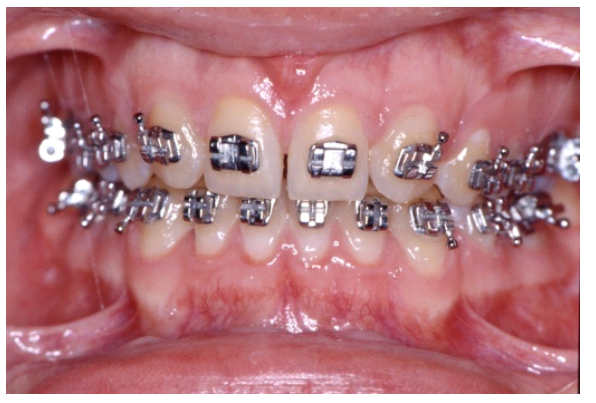

Figure 2. at the End of orthodontic treatment.
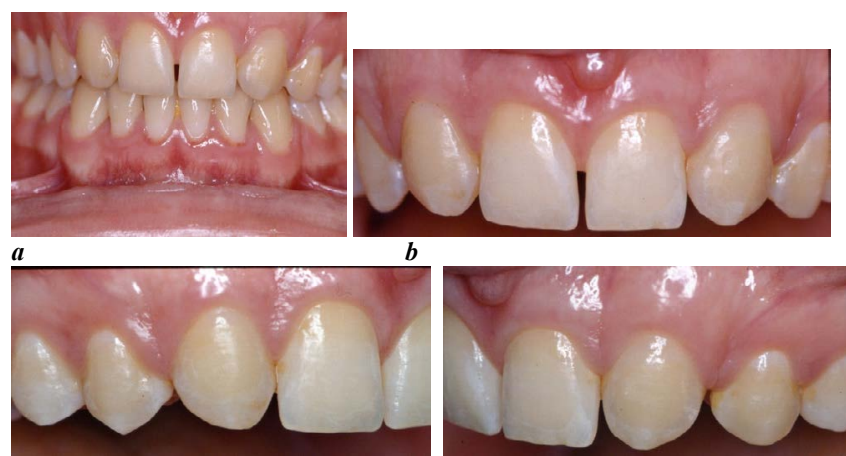

$c$

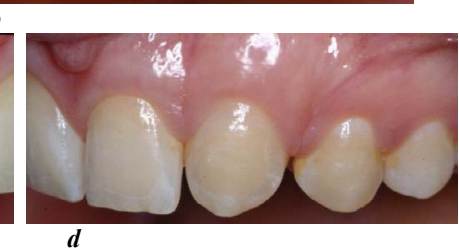

Figure 3a-d. After orthodontic space closure and debonding, a perfect plaque control is required, in order to obtain and maintain a good gingival profile.

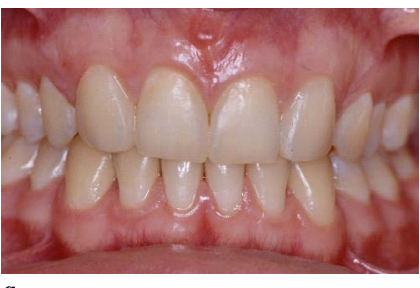

$\boldsymbol{a}$

Figure 4a-b. Two-year follow-up anterior view.

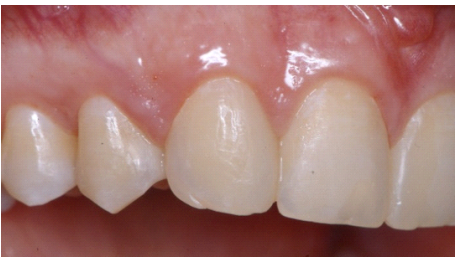

$a$
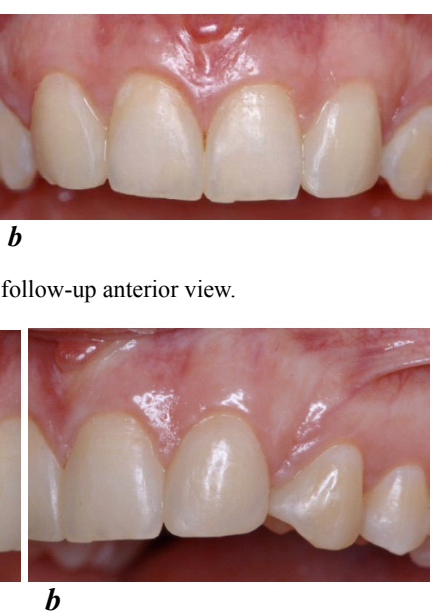

$\boldsymbol{b}$
Figure 5a-b. Two-year follow-up left and right

which means that lifelong prosthetic restorations, that are likely to need repairs or replacements in the future, are avoided. Thus, the total cost of treatment is reduced for patient's benefit $[16,17]$. In addition, clear and natural gingival margin is achieved which will change in synchrony with the patient's own teeth over a lifetime and any change due to the normal aging or for other reasons (mechanical, including tooth brushing, or periodontal) will take on a natural look [18].

The esthetic result of space closure as a treatment option in patients with congenitally missing maxillary lateral incisors is generally preferred by general dentists, orthodontists, combined dental specialists, and laypeople. An interesting point was that a significant percentage of general dentists would restore the missing lateral incisors with implants for esthetic reasons but, even those professionals who felt the missing teeth should be restored, many did not prefer the esthetic result of a restored option $[16,17]$.

In another study, Robertsson et al. evaluated the satisfaction of fifty treated patients with lateral incisor agenesis [19]. They have shown that (a) patients treated by space closure were more satisfied with the treatment results than the prosthesis patients, (b) there was no difference between the 2 groups in prevalence of signs and symptoms of temporomandibular junction (TMJ) dysfunction, and (c)

patients with prosthetic replacements had impaired periodontal health with accumulation of plaque and gingivitis [19].

\section{Disadvantages}

The tendency of the anterior teeth to reopen and relapse, after the orthodontic treatment is completed, is considered as the main disadvantage $[11,18]$. However, this can be overcome with long-term fixed retention with a palatally bonded flexible spiral wire retainer on the palatal surfaces of the six anterior teeth. Another disadvantage of this treatment option is the enameloplasty which is required usually on both the canine and premolar in order to resemble the teeth they substitute $[16,17]$.

Moreover, the color difference between incisors and canines, can cause esthetic problems and requires restoration. In addition, the fact that canine-protected occlusion is not feasible with space closure is considered as a disadvantage by certain authors, due to the stress placed on the premolars [11]. 
Though, long-term occlusal and periodontal studies have shown there is no evidence for establishment of Class I canine relationship and space closure with premolar substitution for canines can lead to an acceptable functional relationship with modified group function on the working side [19].

\section{Tooth-supported restorations approach}

The first step in opening space for a tooth-supported prosthesis or single-tooth implant is to determine how much space is necessary for the missing lateral incisor replacement. There are several methods for doing this. The first method is called the "golden proportion."22 The perceived width of each anterior tooth should have a ratio of 1:1.618 to the tooth adjacent to it $[20,21]$.

The second method is to use the contralateral lateral incisor as a reference, [22] but this method is not suited for patients with missing or peg-shaped contralateral incisors [2,22].

The third method is to conduct a Bolton analysis.24 It involves dividing the sum of the mesiodistal widths of the 6 anterior mandibular teeth by the sum of the mesiodistal widths of the 6 anterior maxillary teeth. The anterior Bolton ratio should be approximately $[1,3,22]$. The Bolton analysis is a quick and reliable way to determine the appropriate space necessary for patients with congenitally missing lateral incisors [22].

The fourth and most predictable guide for determining the ideal replacement space is to construct a diagnostic wax-up. Generally, the maxillary lateral incisor width ranges from 5 to $7 \mathrm{~mm}$. [20,22] (Figures 6 and 7).

\section{Three types of tooth-supported prostheses}

There are 3 basic types of tooth-supported prostheses available today. They are a resin-bonded fixed bridge, a cantilevered fixed bridge,
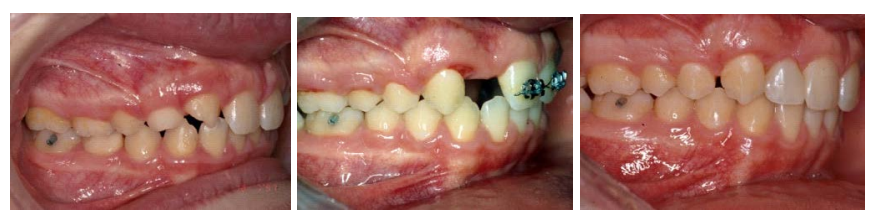

$a$

$b$

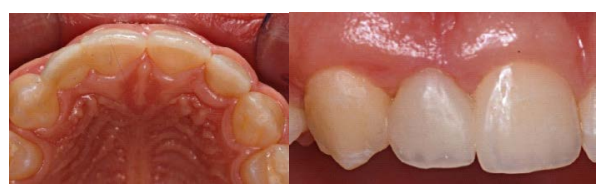

$d$ $e$

Figure 6 a-e. Resin-Bonded to Replace Missing Lateral Incisors.

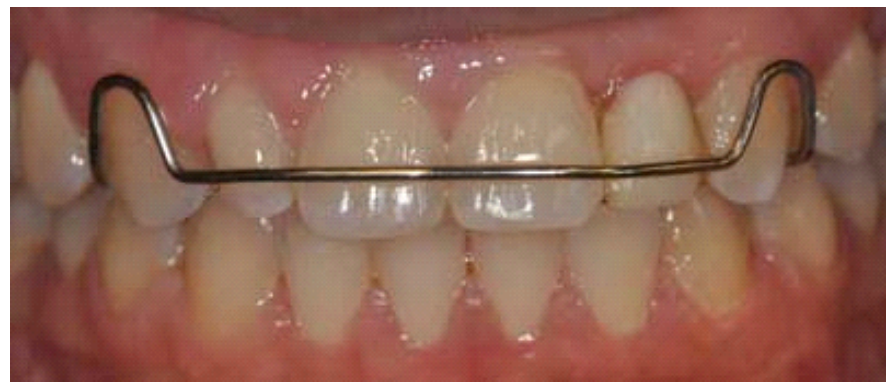

Figure 7. Removable partial denture. and a conventional full-crown fixed bridge. The primary consideration among these treatment options is conservation of tooth structure. Ideally, the treatment of choice should be the least invasive option that satisfies both aesthetic and functional objectives for the patient.

\section{Cantilever bridge}

The second most conservative tooth-supported prothesis designed to replace a congenitally missing lateral incisor is a cantilevered fixed bridge. The success of this type of restoration is not dependent on the amount of proclination or mobility of the abutment teeth. Intracoronal pins provide retention and resistance for a cantilevered bridge; therefore the size of the pulp and its location within the tooth must be evaluated prior to the selection of this type of restoration. The long-term success of the cantilevered fixed bridge depends on management of the effects of occlusion on the pontic(s). Heavy occlusal forces applied on the pontics cause early failures $[20,23]$.

\section{Conventional full-coverage fixed bridge}

The least conservative but sturdiest of all tooth-supported protheses is a conventional full-coverage fixed bridge. A conventional fixed bridge exerts control over the occlusion and occlusal forces [22].

Before a full-coverage fixed bridge is placed, the alignment of the anticipated abutment teeth along a common pathway must be verified. From the frontal view, the long axis of the central incisor and the labial surface of the canine should be parallel.

This allows the prosthodontist to achieve the proper "line of draw" when preparing these teeth. Also, from a lateral perspective, the long axis of the canine and the labial surface of the central incisor must be parallel for proper tooth preparation. The orthodontist must know how to align these teeth according to the specific restorative requirements for the chosen prosthesis. Another consideration is the faciolingual position of the abutment teeth as it relates to palatal tooth preparation and the amount of preparation. The orthodontist can help to reduce the need for tooth preparation by leaving an overjet of approximately 0.5 to $0.75 \mathrm{~mm}[20,22-24]$.

\section{Resin-bonded fixed bridge}

This is the most conservative method for replacing a missing lateral incisor with a tooth-supported prosthesis. The success rate with this type of prosthesis varies widely from $46 \%$ over 11 months to $90 \%$ over 11 years, with the most common form of failure being debonding [22]. The specific criteria for a successful treatment using a resin-bonded fixed bridge include the position, mobility, thickness, and translucency of the abutment teeth as well as the overall occlusion.

Resin-bonded fixed bridges placed in a deep overbite relationship have been shown to have a higher incidence of failure. The ideal anterior relationship for a resin-bonded fixed bridge is a shallow overbite. Another concern regarding tooth position is inclination of the abutment teeth. Abutment teeth with increased inclination are more prone to debonding [20,22-24].

The mobility of the abutment teeth is a contraindication for a resin-bonded fixd bridge. A final area of concern regarding placement of a resin-bonded fixed bridge is occlusal parafunction, which places too much stress on the pontic and subsequently results in prosthesis failure. Abutment teeth that are immobile, moderately thick, and have translucency mainly localized in the incisal one third are ideal candidates for a resin-bonded fixed bridge. A shallow overbite allows maximum surface area for bonding retainers with little or no tooth 
preparation. Each of the above prosthetic methods can be used with a high degree of success if used in the appropriate situation [12,20,22]. Interdisciplinary management of patients with congenitally missing lateral incisors often plays a vital role in the success of the treatment. The combined efforts of the prosthodontist and orthodontist can produce predictable and aesthetic treatment results for congenitally missing lateral incisors $[22,33,24]$.

\section{Implants approach}

With lateral incisor agenesis and available space, implants are usually the treatment of choice. Implants are a favorable option because no adjacent tooth is prepared for restorations, and implants have a success rate of $90 \%$ over 10 years [25]. Pre-implant orthodontics must leave adequate room for the implant between the adjacent roots as well as sufficient crown space. This can be achieved by using the golden proportion, the contralateral lateral incisor, a Bolton analysis, or a diagnostic wax-setup. Generally the lateral incisor site should be $5-7 \mathrm{~mm}$. Space between the roots of the adjacent teeth and the implant can be no less $0.75 \mathrm{~mm}$, with $1.5-2 \mathrm{~mm}$ space between the adjacent crowns and implant head [26] Implants must be placed after growth cessation due to the continuing vertical growth of the jaws. If growth has not stopped, this can lead to infraocclusion of the implant with an unesthetic gingival architecture. On average boys finish growth at 21 years of age and girls at 17 years $[1,2,5,10]$. After orthodontics, the adjacent roots must be maintained out of the edentulous site, and the alveolar ridge may need bone grafting in the future if the ridge narrows. The lateral incisor space will also need a temporary pontic, which is often built into a retainer or a RMB. If the implant is placed too labially, the thin buccal bone can resorb and the gingiva can appear gray in color. Poor soft tissue management can also lead to loss of papillary esthetics; the papilla distal to the lateral incisor implant can be particularly difficult to fill in the embrasure space [22] (Figure 8).
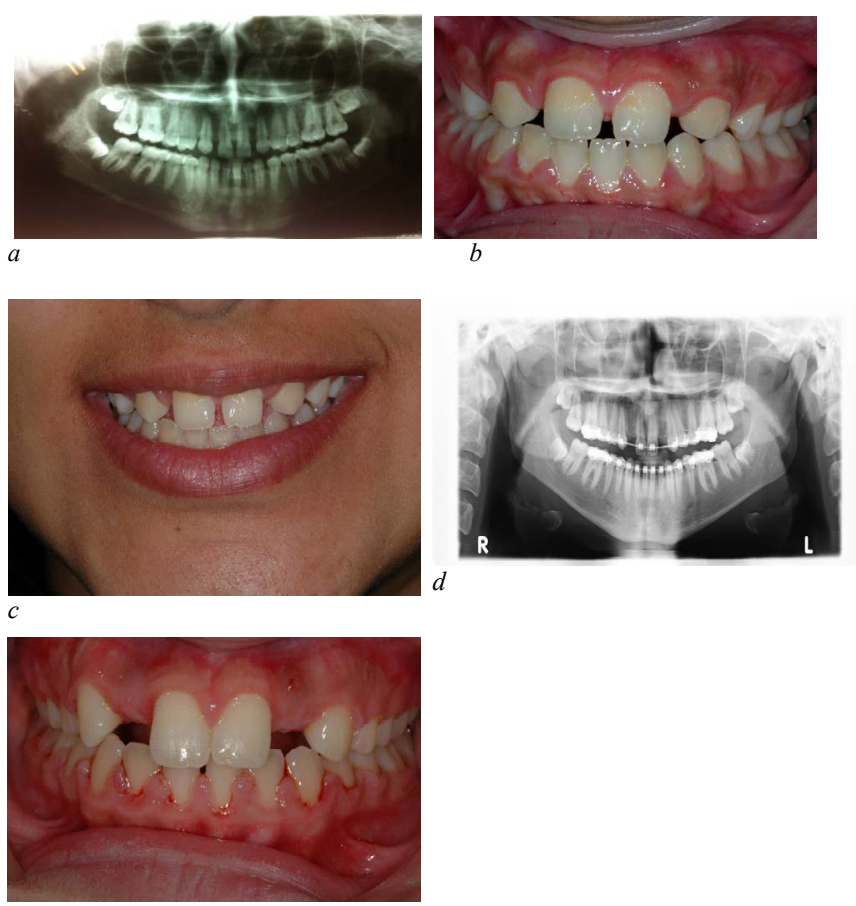

$e$

Figure 8 a-c. Pre-orthodontics. d-e. Pre-operative view.
Several criteria have to be considered before placing a single tooth implant in adolescents:

\section{Time of implant placement}

2. Development of a proper implant site

3. Space needed coronally

4. Space needed apically

5. Height of gingiva

6. Retention of space needed before implant placement

Generally, implants must not be placed until the patients have completed their facial growth and the majority of their tooth eruption. As the face grows and the mandibular rami lengthen, teeth must erupt to remain in occlusion [27].

However, the implant behaves like an ankylosed tooth and will not follow the changes of the alveolar processes due to the eruption of adjacent teeth [28].

This may result in clinical infraocclusion of the implant-supported crown and cause a discrepancy in the occlusal plane and between the gingival margins of the implant and the adjacent natural teeth $[27,28]$ Thus, evaluation of the completion of facial growth by cephalometric radiographs must be done and subsequently, the patient should be informed for the optimal time of implant placement $[10,12,26]$. However, even mature adults can exhibit major vertical steps after anterior restorations with implants to the same extend as adolescents [29] (Figure 9).

To achieve a predictable and esthetically satisfying implant outcome, it is crucial to have a properly developed implant site. The buccolingual dimension of the alveolar ridge has to be wide enough to allow a surgeon to place the implant in a correct 3-D position [30-32].

If the buccolingual dimension is insufficient, a bone graft may be necessary. An ideal method to develop a proper width of the alveolar ridge can be achieved if the canine erupts next to the central incisor. The buccolingual width of the canine creates a sufficient width of the ridge when erupting. After eruption, the canine can be distalized orthodontically and, therefore, establish a proper buccopalatal width of the alveolar ridge. Studies have demonstrated that if an implant site is developed with this kind of orthodontic guided tooth movement, the buccopalatal width remains stable. Distalizing may need to be done with bodily movement to develop adequate space between the roots. If a panoramic radiograph reveals that the permanent canine is apical to the primary canine, the extraction of the primary lateral incisor may be considered to guide the eruption of the permanent canine toward the central incisor $[1,22,32]$. This (as explained previously) is favorable for developing a proper implant site. Otherwise, it may not

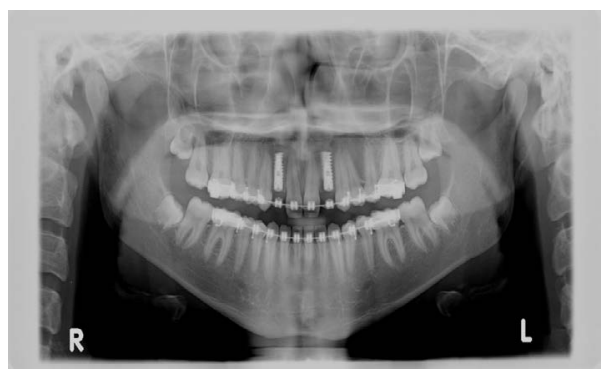

Figure 9. Post-operative radiograph. 
be possible to guide the eruption of the canine near the central incisor, the osseous ridge will not fully develop, and the buccopalatal width will be insufficient for a proper implant placement. In these cases, it is necessary to perform a bone graft before or at the time of implant placement to achieve a sufficient dimension of the alveolar ridge [1-5].

The amount of space needed for the implant and crown is generally determined by the contralateral lateral incisor. However, if both lateral incisors are missing or the contralateral one is peg-shaped, the amount of space should be determined by one of the methods below:

\section{The golden proportion}

\section{The Bolton analysis}

\section{A diagnostic wax-up}

Generally, the adequate coronal space should be no less than 6,3 $\mathrm{mm}$ where as the interradicular space no less than $5,7 \mathrm{~mm}$. [33] At least, $1 \mathrm{~mm}$ between of the implant and adjacent roots is desirable as it is cited that narrower distances between them are more likely to show a reduction in bone height over time. In addition, fixed retention is suggested rather than removable appliances to prevent relapse [26].

When the orthodontist opens space for the missing lateral incisor with fixed appliances, he should be very careful so the central incisor and the canine are moved by... and not to tip apart, because this is likely to make implant placement impossible. Thus, the orthodontist must confirm the ideal root position with a periapical or a panoramic radiograph, before the fixed appliances are removed $[10,12,26]$.

In certain patients, it may be impossible to achieve acceptable interradicular spacing, even though the coronal spacing may be ideal. Particularly, in a patient with a Class III tendency malocclusion who requires proclination of the maxillary central incisors, when the crowns are tipped labially, the roots tend to converge toward each other resulting in a "wagonwheel" effect. In such cases, an alternative restoration option is required [34-36].

Implants can be placed in women at approximately age 17 and in men at approximately age 21 . At these ages, craniofacial growth is generally completed. Treatment of these patients should start before this age because development of the alveolar ridge has to be achieved and coronal space and interradicular space has to be created. That leads to a period of time when space maintenance may have to be provided for a patient, if craniofacial growth is not yet completed. The temporization and stabilization depends on the waiting time until the implant may be placed. If patients are ready for implant placement in a couple of months, a removable retainer, such as a Hawley retainer or an Essix retainer with a built-in prosthetic tooth, can be used. If patients have to wait 1 or 2 years before completion of growth is achieved, a temporary resin-bonded bridge is the more favorable option [34-37].

\section{Advantages of implant approach}

\section{-No adjacent teeth have to be prepared}

-Successful osseointegration of implants

\section{Disadvantages of implant approach}

-Esthetic outcome may be worse than orthodontic approach and/ or prosthetic approach (long term)

-Needs perfect team play between orthodontist, oral surgeon, and prosthodontist or result could be compromised

-Apical migration of gingival and bone if traditional implants are used-additional need of grafting overtime to obtain esthetic results (Figure 10).

\section{Autotransplantation approach}

At present, autotransplantation of teeth has evolved into a viable treatment option for replacing missing teeth, since successfully transplanted teeth have been proved to function as totally normal teeth [38] Autotransplantation of teeth ensures that alveolar bone volume is maintained, due to physiological stimulation of the periodontal ligament. Moreover, successful tooth transplantation offers improved esthetics, arch form, dentofacial development, mastication, speech and arch integrity. Autotransplants, unlike restorative prosthetic units, also provide proprioception during function and can be used in the growing patient. Finally, the total treatment cost is normally lower in comparison to alternative prosthetic restorative and/or orthodontic treatment options $[39,40]$.

Further, in cases of congenital absence of permanent teeth, replacement with autotransplanted teeth guarantees that the alveolus will not atrophy, especially when the patient is still at a growing age. Successful tooth transplantation in young individuals also facilitates dentofacial development, mastication and speech, along with maintenance of the attached gingivae, in a natural shape and level. Even in the case of failure after a certain number of years, the maintenance of alveolar bone volume until then is essential. This is especially the case when a dental implant has been chosen as an alternative restorative solution [40-44].

The optimal time for the autotransplantation of premolars to the maxillary incisor area is when the development of the roots of the premolars has reached two-thirds to three-fourths of the final root length. If the timing is right, which means patients are approximately 9 to 12 years of age, the periodontal healing is better than $90 \%[38,39]$.

Root growth continues after the autotransplantation and the teeth maintain their capacity for functional adaption; endodontic treatment is most of the time not necessary after treatment. Next to timing, the surgical technique for tooth transplant is of great importance for the success of the treatment, which means that any damage of the periodontal ligament has to be avoided; otherwise, alkalosis may occur.

The long-term results for autotransplantation are impressive. Czochrowska et al. found, in their long-term studies, including 33 transplanted premolars with a mean of 26.4 years, a survival rate of 90\% [45-47].

Additional orthodontic treatment after autotransplantation is possible, because a normal periodontal ligament is established. Waiting 3 to 4 months is recommended before any orthodontic treatment is started $[38,39]$. The premolars are usually reshaped and build up with composite in the beginning; later on, when an ideal result is achieved,
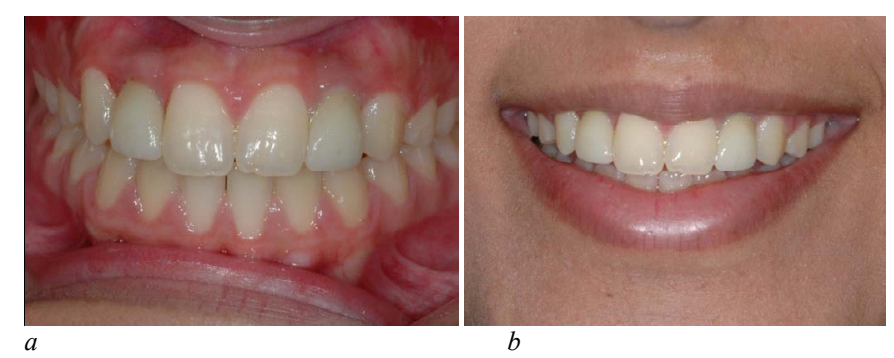

Figure 10 a-b. Zirconia crowns cemented. 
the composites can be replaced with porcelain veneers.

\section{Advantages of autotransplantation approach}

-Biologic approach

-Creates alveolar bone

-Periodontal membrane

-Adjustable alveolar bone

-Periodontal membrane after surgery with orthodontics

-Normal interdental papilla

-Good long-term results.

\section{Disadvantages of autotransplantation approach}

\section{-Experienced surgeon necessary}

-Very technique sensitive

-Age limitation, 9-12years

The transplanted tooth can serve and function as a normal tooth. Therefore, in addition to improved esthetics and mastication, successful tooth transplantation offers arch space maintenance and preserves the volume and morphology of the alveolar bone. The cost is also considerably reduced in comparison to advanced treatment options such as dental implants and/or prosthetic replacements; moreover, it can be performed as a single-step surgical procedure [48-50].

\section{Conclusions}

The successful restorative treatment depends on interdisciplinary treatment planning, especially if preprosthetic orthodontic tooth alignment is required similar to case report. Dental implants are a treatment of choice for most patients with congenitally missing laterals. An implant will preserve adjacent tooth structure and alveolar bone and provide esthetics and function. Golden proportion can play a key role in such cases by providing reference for space consideration.

\section{References}

1. Abu-Hussein M, Abdulgani A, Watted N, Zahalka M (2015) Congenitally Missing Lateral Incisor with Orthodontics, Bone Grafting and Single-Tooth Implant: A Case Report. J Dent Med Sci 14: 124-130.

2. Abdulgani A, Kontoes N, Chlorokostas G, Abu-Hussein M (2015) Interdisciplinary Management Of Maxillary Lateral Incisors Agenesis With Mini Implant Prostheses: A Case Report. J Dent Med Sci 14: 36-42.

3. Abusalih A, Ismail H, Abdulgani A, Chlorokostas G, Abu-Hussein M (2016) Interdisciplinary Management of Congenitally Agenesis Maxillary Lateral Incisors: Orthodontic/Prosthodontic Perspectives. J Dent Med Sci 15: 90-99.

4. Abu-Hussein M, Watted N, Abdulgani A, Borbély B (2015) Modern Treatment for Congenitally Missing Teeth: A Multidisciplinary Approach; International Journal of Maxillofacial Research 1: 179-190.

5. Abu-Hussein M, Chlorokostas G, Watted N, Abdulgani A, Jabareen A (2016) PreProsthetic Orthodontic Implant for Management of Congenitally Unerupted Lateral Incisors - A Case Report. J Dent Med Sci 15: 99-104.

6. Abu-Hussein M, Watted N, Hegedus V, Péter B (2015) Congenitally Missing Upper Laterals. Clinical Considerations: Orthodontic Space Closure 1: 1-6.

7. Rendon J (2005) Effect of congenitally missing lateral incisors on the eruption and impaction of the maxillary canine: Saint Louis University.

8. Araújo EA, Oliveira DD, Araújo MT (2006) Diagnostic protocol in cases of congenitally missing maxillary lateral incisors. World J Orthod 7: 376-388. [Crossref]

9. Abu-Hussein M, Watted N, Yehia M, Proff P, Iraqi F (2015) Clinical Genetic Basis of Tooth Agenesis. J Dent Med Sci 14: 68-77.
10. Kokich VO Jr, Kinzer GA (2005) Managing congenitally missing lateral incisors. Part I: Canine substitution. J Esthet Restor Dent 17: 5-10. [Crossref]

11. Sabri R (1999) Management of missing maxillary lateral incisors. $J$ Am Dent Assoc 130: 80-84. [Crossref]

12. Kokich VG, Spear FM (1997) Guidelines for managing the orthodontic-restorative patient. Semin Orthod 3: 3-20. [Crossref]

13. Chu SJ, Tan JH, Stappert CF, Tarnow DP (2009) Gingival zenith positions and levels of the maxillary anterior dentition. J Esthet Restor Dent 21: 113-120. [Crossref]

14. Chu SJ (2007) Range and mean distribution frequency of individual tooth width of maxillary anterior dentition. Pract Proced Aesthet Dent 19: 209-15. [Crossref]

15. Zachrisson BU, Mjör IA (1975) Remodeling of teeth by grinding. Am J Orthod 68: 545-553. [Crossref]

16. Armbruster PC, Gardiner DM, Whitley JB, Flerra J (2005) The congenitally missing maxillary lateral incisor. Part 1:Esthetic judgment of treatment options. World J Orthod 6: 369-375. [Crossref]

17. Armbruster PC, Gardiner DM, Whitley JB, Flerra J (2005) The congenitally missing maxillary lateral incisor. Part 2: Assessing dentist's preferences for treatment. World $J$ Orthod 6: 376-381. [Crossref]

18. Rosa M, Zachrisson BU (2007) Integrating space closure and esthetic dentistry in patients with missing maxillary lateral incisors. J Clin Orthod 41: 563-573. [Crossref]

19. Robertsson S, Mohlin B (2000) The congenitally missing upper lateral incisor. A retrospective study of orthodontic space closure versus restorative treatment. Eur $J$ Orthod 22: 697-710 [Crossref]

20. Abu-Hussein M, Watted N, Abdulgani A, Kontoes N (2015) Prosthodontic-Orthodontic Treatment Plan with Two-UnitCantilevered Resin-Bonded Fixed Partial Denture. IOSR-JDMS 14: 131-136.

21. Abu-Hussein M, Chlorokostas G, Watted N, Abdulgani A, Jabareen A (2016) PreProsthetic Orthodontic Implant for Management of Congenitally Unerupted Lateral Incisors - A Case Report. J Dent Med Sci 15: 99-104.

22. Abu-Hussein M, Watted N, Abdulgani A, Borbély B (2015) Modern Treatment for Congenitally Missing Teeth: A Multidisciplinary Approach. Int J maxillofacial res 1: 179-190.

23. Bishop K, Addy L, Knox J (2007) Modern restorative management of patients with congenitally missing teeth: 3 . Conventional restorative options considerations. Dent Update 34: 30-38. [Crossref]

24. Mai A, Azzaldeen A, Nezar W, Chlorokostas G, Muhamad AH (2016) Extraction and Immediate Implant Placement with Single-StageSurgical Procedure: Technical Notes and a Case Report. J Dent Med Sci 15: 95-101.

25. Zachrisson BU (1978) Improving orthodontic results in cases with maxillary incisors missing. Am J Orthod 73: 274-289. [Crossref]

26. Kokich VG (2004) Maxillary lateral incisor implants: planning with the aid of orthodontics. J Oral Maxillofac Surg 62: 48-56. [Crossref]

27. Lecture KV (2009) Missing maxillary lateral incisors: The agony and ecstasy of implant replacement. AAO Meeting. Boston, MA.

28. Thilander B (2008) Orthodontic space closure versus implant placement in subjects with missing teeth. J Oral Rehabil 35 Suppl 1: 64-71. [Crossref]

29. Odman J, Gröndahl K, Lekholm U, Thilander B (1991) The effect of osseointegrated implants on the dento-alveolar development. A clinical and radiographic study in growing pigs. Eur J Orthod 13: 279-286. [Crossref]

30. Bernard JP, Schatz JP, Christou P, Belser U, Kiliaridis S (2004) Long-term vertical changes of the anterior maxillary teeth adjacent to single implants in young ?a? mature adults. A retrospective study. J Clin Periodontol 31: 1024-1028. [Crossref]

31. Muhamad AH, Azzaldeen A, Nezar W, Mohammed Z (2015) Esthetic Evaluation of Implants Placed after Orthodontic Treatment in Patients with Congenitally Missing Lateral Incisors. J Adv Med Dent Scie Res 3: 110-118.

32. Abdulgani M, Abdulgani Az, Abu-Hussein M (2016) Two Treatment Approaches for Missing Maxillary Lateral Incisors: A Case. J Dent Med Sci 15: 78-85.

33. Abu-Hussein M, Watted N, Abdulgani A (2016) Managing congenitally missing lateral incisors with single tooth implants. Dent Oral Craniofac Res 2: 318-324.

34. Olsen TM, Kokich VG (2010) Postorthodontic root approximation after opening space for maxillary lateral incisor implants. Am J Orthod Dentofacial Orthop 137:158 [Crossref] 
35. Abu-Hussein M, Georges C, Watted N, Azzaldeen A (2016) A Clinical Study Resonance Frequency Analysis of Stability during the HealingPeriod. Int J Oral Craniofac Sci 2: 065-071.

36. Muhamad AH, Nezar W, Azzaldeen A (2016) Esthetic Management of Congenitally Missing Lateral Incisors with Single Tooth Implants: A Case Report. J Dent Med Sci 15: 69-75.

37. Kinzer GA, Kokich VO Jr (2005) Managing congenitally missing lateral incisors. Part III: single-tooth implants. J Esthet Restor Dent 17: 202-210. [Crossref]

38. Muhamad AH, Azzaldeen A (2012) Autotransplantation of Tooth in Children with Mixed Dentition. Dentistry 2: 149.

39. Abu-Hussein M, Watted N, Abdulgani M, Abdulgani Az (2016) Tooth Autotransplantation; Clinical Concepts. J Dent Med Sci 15: 105-113 .

40. Muhamad AH, Azzaldeen A (2013) Auto transplantation of tooth in mixed dentition- A review. Int J Dent Clinics 5: 20-23.

41. Abu-Hussein M, Watted N, Abdulgani A (2014) Replantation of Avulsed Permanent Anterior Teeth: A Case Report. RRJDS 2: 43-52.

42. Abu-Hussein M, Azzaldeen A (2016) Intentional replantation of maxillary second molar; case report and 15-year follow-up. J Dent Med Sci 15: 67-73 .
43. Abu-Hussein M, Nezar W, Azzaldeen A, Abdulgani M (2016) Prevalence of Traumatic Dental Injury in Arab Israeli Community. J Dent Med Sci 15: 91-98 .

44. Abu-Hussein M, Aspasia S, Azzaldeen A (2013) Eight-year follow-up of successfu intentional replantation roots $3: 28-31$.

45. Czochrowska EM, Stenvik A, Album B, Zachrisson BU (2000) Autotransplantation of premolars to replace maxillary incisors: a comparison with natural incisors. $\mathrm{Am} \mathrm{J}$ Orthod Dentofacial Orthop 118: 592-600. [Crossref]

46. Abu-Hussein M, Watted N, Abdulgani M, Abdulgani Az (2016) Tooth Autotransplantation; Clinical Concepts. J Dent Med Sci 15: 105-113

47. Muhamad AH, Azzaldeen A (2012) Autotransplantation of Tooth in Children with Mixed Dentition. Dentistry 2: 149.

48. Abu-Hussein M, Watted N, Abdulgani A (2015) Autogenous Tooth Transplantation Reality Or Not. Int J Dent Health Sci 2: 722-730

49. Abu-Hussein M, Watted N (2016) Maxillary Midline Diastema - Aetiology And Orthodontic Treatment- Clinical Review. J Dent Med Sci 15: 116-130

50. Abu-Hussein M, Georges C, Watted N, Azzaldeen A (2016) A Clinical Study Resonance Frequency Analysis of Stability during the Healing Period. Int J Oral Craniofac Sci 2: 065-071.

Copyright: (C2016 Watted N. This is an open-access article distributed under the terms of the Creative Commons Attribution License, which permits unrestricted use, distribution, and reproduction in any medium, provided the original author and source are credited. 